\title{
Investigation of controllable multi electrode based FES (functional electrical stimulation) system for restoration of grasp- preliminary study on healthy individuals
}

\author{
Vinil T C, Suresh Devasahayam \\ Dept. of Bioengineering \\ Christian Medical College \\ Vellore, India \\ Email: surdev@cmcvellore.ac.in
}

\author{
George Tharion, Naveen B P \\ Dept. of Physical Medicine and Rehabilitation \\ Christian Medical College \\ Vellore, India
}

\begin{abstract}
Functional electrical stimulation applied via surface electrode can be used for hand rehabilitation particularly for enabling grasp in patients with stroke or spinal cord injury. The use of multi-pad electrode and multi-channel electrical stimulator based improve the effectiveness of conventional FES. Such a system consists of a multi-pad surface electrode and a matching multi-channel stimulator. This system will allow the targeting of motor neurons which activate muscle groups to produce corresponding functional movements of the hand. This paper presents our study on normal subjects to quantify the movement resulting from stimulation of electrodes spatially distributed around the forearm. The device was tested on four healthy subjects and the results show that multi-pad electrode provide desired amount of selectivity and can be used for generating functional grasp. The results also show that the effect of stimulation varies from person to person reflecting inters subject anatomical variability.
\end{abstract}

Index Terms-Functional electrical stimulation, neuroproshthesis, multi-pad electrode, paralysed hand.

\section{INTRODUCTION}

It is often impossible for patients who have suffered spinal cord injury or stroke to use their limbs despite the presence of an intact peripheral nervous system and intact musculoskeletal system $[1,2]$. Functional electrical stimulation (FES) is an important technique in the field of assistive technologies which can provide artificial control over the peripheral nervous system that is compromised after stroke or spinal cord injury $[1,2]$. FES is a method of applying a few milli-amperes of electrical currents to the body to activate underlying nerves and restore impaired function. An FES based neuroprosthesis applies electrical stimulation to the motor branches of the peripheral nerve to activate paralysed muscles to produce contraction. FES has been used for rehabilitation purposes for many years [3, 4]. FES based grasping systems have existed in research settings for the last few decades. Various combinations of FES systems have been in use for regaining grasp and release function. One example is the use of FES with splint for grasp [8].

Common problems in many existing FES systems are 1.) Insufficient selectivity, 2.) Discomfort, 3.) Fast fatigue. Many of these problems can be ameliorated by the use of multi-pad electrode instead of a large single electrode [9]. The suggestion to use multi-pad electrodes was introduced in parallel with the development of multi-channel electrical stimulator for patients with tetraplegia or stroke [1, 9]. By using multi-pad electrodes the electrical charge can be distributed so as to cause less discomfort compared to large surface electrodes $[1,9]$.

The application of multiple small surface electrode pads results in controlled localization of current and avoid spreading of current across the motor neurons of near muscles. This enables controlled charge distribution which is essential for the functional outcome.

We have developed a FES grasping system consisting of multi-pad electrodes and multi-channel electrical stimulator, with user interface for selecting various stimulation parameters and electrode shape configuration. This paper will focus on the outcome of our experiments done on normal subjects.

\section{METHODS}

The apparatus used in our experiments comprised a multichannel stimulator and a wearable multi-electrode pad. The movement produced by the stimulation protocol was captured using a video camera and later quantized from the video frames. Individual electrodes synchronously will improve the functional outcome as well as will postpone muscle fatigue. 

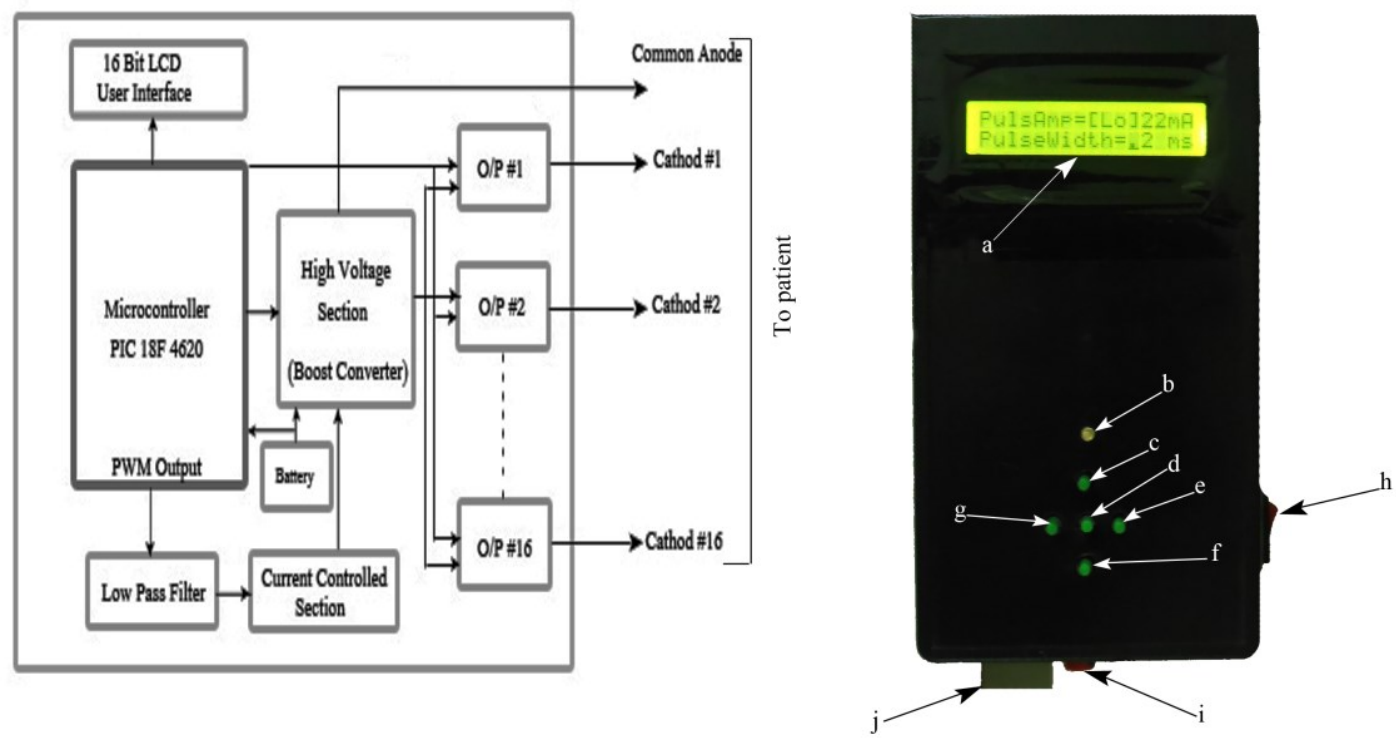

Fig 1 Multi-channel stimulator: block diagram (left panel) and front panel of the stimulator (right panel). The letters at the right panel represents a) LCD display, b)Indication LED, c)Increment button, d)Trigger button,e)Parameter selection button,f)Decrement button,g)Parameter selection button, h) Power ON switch, i) Anode connector, i)Cathode connector

A corresponding sixteen electrode array band was made using silver discs sewed on an elastic fabric backing.

The electrode band was connected to the stimulator using a flexible flat cable.

\section{A. Stimulation hardware}

The stimulator is a constant current stimulator, as current pulses are independent of contact electrode impedance. Our stimulator uses an 8 bit microcontroller PIC18F4620 (Microchip). The circuit needs a high voltage source to be able to inject desired current level to the skin (for electrode impedances up to $4 \mathrm{~K}$ Ohms). The output stage or current controlled stage of the stimulator is limited to $200 \mathrm{~V}$. This voltage is generated by an on-demand highvoltage generator. There are sixteen independent constant current output sections corresponding to sixteen channels.

The user interface comprises a $2 \times 16$ character LCD display and 5 buttons. Four of the buttons are used for navigation through a menu list to select stimulation parameters such as pulse amplitude, pulse width, frequency, duration and selection of electrodes. Once the stimulation parameters and electrode set (any combination of the 16 electrodes) have been selected, stimulation can be initiated using the centre button
The stimulator is powered using four $\mathrm{AA} \mathrm{Ni}-\mathrm{MH}$ rechargeable batteries.

\section{B. Multi-pad electrode}

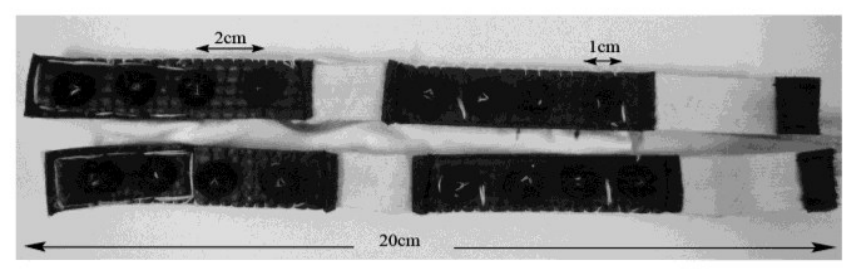

Fig 2: Custom made multi-pad electrode

Our custom made sixteen electrode bands is shown in fig 2. The electrode band is made of Rexine strips and elastic bands stitched together to form a stretchable band. In the Rexine strips cavities are cut to accommodate silver disc electrodes. Over each silver disc electrode a disc of conductive foam is stitched. (Four small holes in each silver disc allow the foam to be stitched to the electrode). This conductive foam when soaked with saline provides good electrical conductivity between the electrode and the tissue. This electrode band forms the multi-electrode cathode. A single band electrode forms the common anode. 


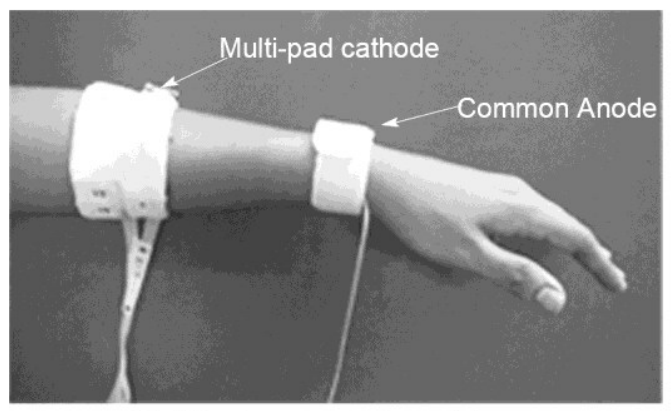

(a) Hand at normal position

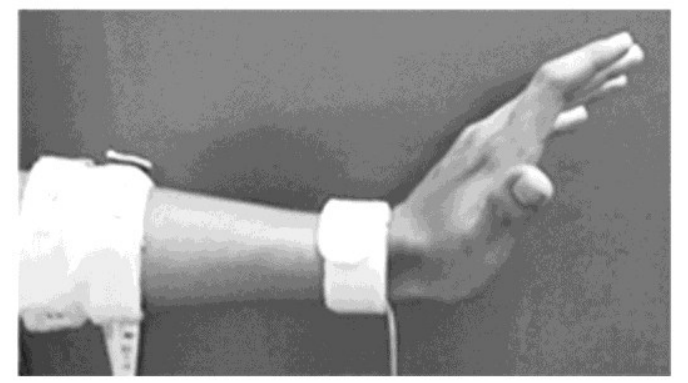

(b) Wrist extension

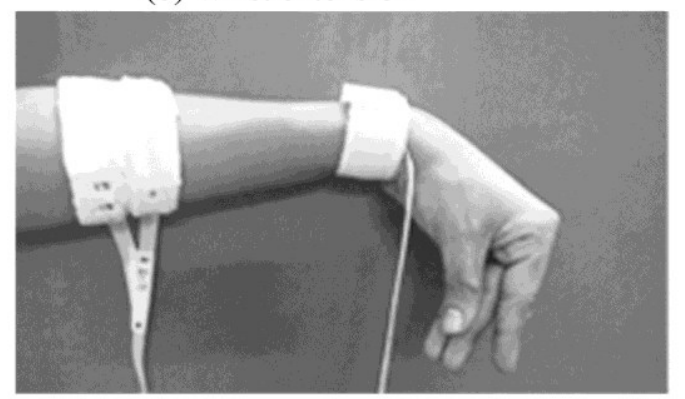

(c) Wrist flexion

Fig 3: Wrist movement on stimulating electrode 2 (extension), electrode 6 (flexion)

\section{Subjects and Experiment}

Our multi-electrode system was tested on four healthy individuals. Written informed consent approved by local Ethics Committee was obtained. The multi-electrode cathode was wrapped around the forearm and held with Velcro. The common anode was placed at the wrist. Stimulating parameter values were; current amplitude of $20 \mathrm{~mA}$, pulse width of $0.2 \mathrm{mS}$, and frequency of $16 \mathrm{~Hz}$. Stimulation was administered through each of the electrode one at a time for duration of 2seconds each. The motor effects of stimulation were measured using a video camera, and by analyzing the video data, the direction and extent of movement, force was determined.

The measurement of extent of movement was taken with a resolution of $15^{\circ}$.

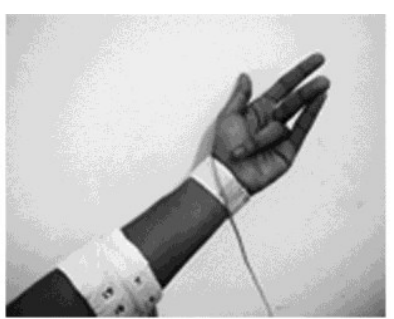

(a)

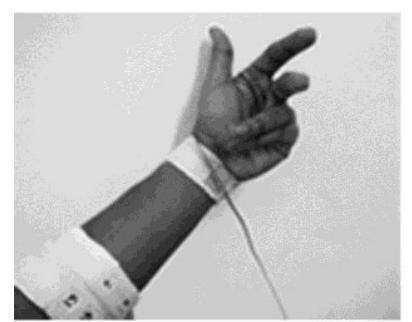

(c)

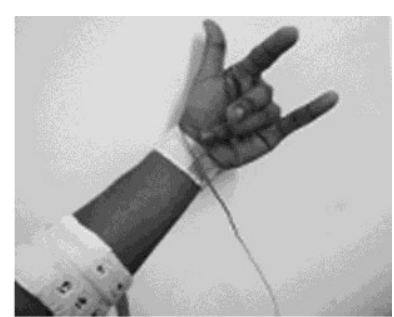

(b)

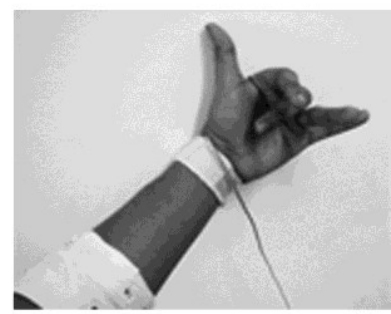

(d)
Fig 4: Individual motion on fingers, (a) middle fingers, (b) ring+middle, (c)small+ring, (d) index+middle

\section{RESULTS AND CONCLUSION}

In this study we recorded individual movements of fingers in terms of extension and flexion of individual digits and wrist using stimulation pulse trains of $16 \mathrm{~Hz}, 0.2 \mathrm{~ms}$ for duration of 2 seconds on each electrode. The movement elicited by results represented in figure 3 and 4 .

Fig 5 shows the results of stimulation of each electrode at Meta carpal phalangeal (MCP) joint, and Proximal inter phalangeal (PIP) joint.

The range of movement at the various joints during individual electrode stimulation depends on the spatial location of the nerves under the electrodes.

During pronation and supination of the forearm, the position of the tissue under the skin changes and that can affect the stimulation. This is an important issue for the future improvement of the device. Our finding suggests that it is possible to form a pattern of electrodes corresponding to produce accurate movement for grasping. However this pattern will vary person to person depends upon their inter subject anatomical variance.

Our FES system for restoration of grasp tested on four healthy individuals. The possibility of spatial reconfiguration of stimulation sites without the movement of electrodes contributes effective way of reproducing functional movements in spinal cord injury or stroke 
patients. In order to characterise the efficacy of our system, more studies have to be done on patients.
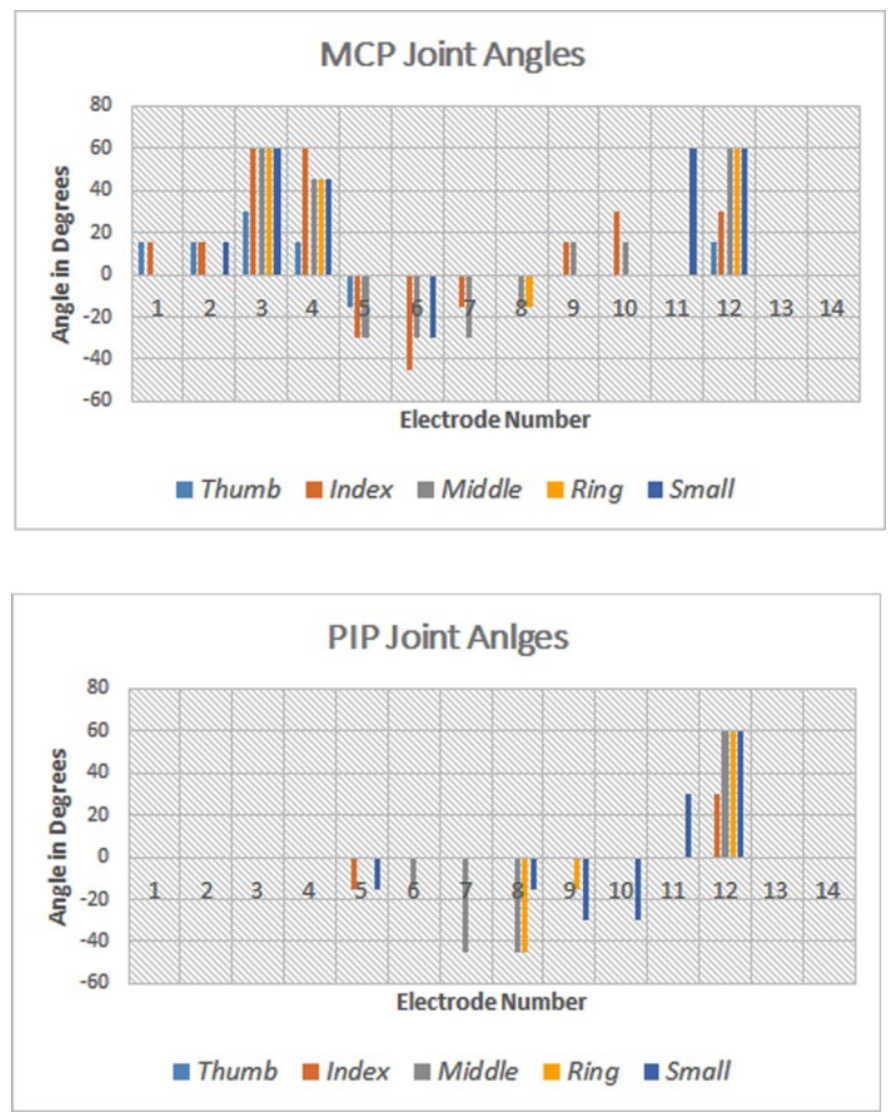

Fig 5: Mean values of joint angles at MCP and PIP measured in one healthy individual when activating all 14 pads one by one

\section{REFERNCE}

[1] N. M. Malesević, L. Z. Popović, L. Schwirtlich, and D. B. Popović, "Distributed low-frequency functional electrical stimulation delays muscle fatigue compared to conventional stimulation.," Muscle Nerve, vol. 42, no. 4, pp. 556-62, Oct. 2010.

[2] T. Watanabe, Y. Tagawa, E. Nagasue, and N. Shiba, "Surface electrical stimulation to realize task oriented hand motion," Conf. Proc. Int. Conf. IEEE Eng. Med. Biol. Soc., vol. 2009, no. 9, pp. 662-665, 2009.

[3] D. B. Popović and M. B. Popović, "Automatic determination of the optimal shape of a surface electrode: selective stimulation.," J. Neurosci. Methods, vol. 178, no. 1, pp. 174-81, Mar. 2009.

[4] A. Popović-Bijelić, G. Bijelić, N. Jorgovanović, D. Bojanić, M. B. Popović, and D. B. Popović, "Multi-field surface electrode for selective electrical stimulation.," Artif. Organs, vol. 29, no. 6, pp. 448-452, 2005.

[5] N. M. Malesević, L. Z. Popović, L. Schwirtlich, and D. B. Popović, "Distributed low-frequency functional electrical stimulation delays muscle fatigue compared to conventional stimulation.," Muscle Nerve, vol. 42, no. 4, pp. 556-62, Oct. 2010.

[6] G. J. Snoek, M. J. IJzerman, F. a In T Groen, T. S. Stoffers, and G. Zilvold, "Use of the NESS handmaster to restore handfunction in tetraplegia: clinical experiences in ten patients.," Spinal cord Off. J. Int. Med. Soc. Paraplegia, vol. 38, no. 4, pp. 244-249, 2000.

[7] N. Malesevic, L. Popovic, G. Bijelic, and G. Kvascev, "Muscle twitch responses for shaping the multi-pad electrode for functional electrical stimulation," J. Autom. Control, vol. 20, no. 1, pp. 53-58, 2010.

[8] Schill, O.; Rupp, R.; Pylatiuk, C.; Schulz, S.; Reischl, M., "Automatic adaptation of a self-adhesive multielectrode array for active wrist joint stabilization in tetraplegic SCI individuals," Science and Technology for Humanity 2009 IEEE Toronto International Conference, vol., no., pp.708,713, 26-27 Sept. 2009

[9] Prochazka, M. Gauthier, M. Wieler, and Z. Kenwell, "The bionic glove: an electrical stimulator garment that provides controlled grasp and hand opening in quadriplegia.," Arch. Phys. Med. Rehabil., vol. 78, no. 6, pp. 608-614, 1997. 